\title{
Reactive Oxygen Species (ROS)-Responsive Nanomedicine for Solving Ischemia-Reperfusion Injury
}

\author{
Weiyu Chen ${ }^{1,2}$ and Deling $\mathrm{Li}^{3 *}$ \\ ${ }^{1}$ The Fourth Affiliated Hospital, Zhejiang University School of Medicine, Yiwu, China, ${ }^{2}$ Molecular Imaging Program at \\ Stanford, Department of Radiology, Stanford University, Stanford, CA, United States, ${ }^{3}$ Department of Neurosurgery, Beijing \\ Tiantan Hospital, Capital Medical University, Beijing, China
}

\section{OPEN ACCESS}

Edited by:

Dawei Jiang,

Huazhong University of Science and

Technology, China

Reviewed by:

Zhilei Ge,

Shanghai Jiao Tong University, China Weijun Wei,

University of Wisconsin-Madison,

United States

*Correspondence:

Deling $\mathrm{Li}$

ttyyneuroli@126.com

Specialty section:

This article was submitted to Nanoscience,

a section of the journal

Frontiers in Chemistry

Received: 25 June 2020

Accepted: 15 July 2020

Published: 21 August 2020

Citation:

Chen W and Li D (2020) Reactive Oxygen Species (ROS)-Responsive Nanomedicine for Solving

Ischemia-Reperfusion Injury.

Front. Chem. 8:732.

doi: 10.3389/fchem.2020.00732
Ischemia-reperfusion injury (IRI) is a severe condition for most organs, which could occur in various tissues including brain, heart, liver, and kidney, etc. As one of the major hazards, reactive oxygen species (ROS) is excessively generated after $I R I$, which causes severe damage inside tissues and further induces the following injury via inflammatory response. However, current medical strategies could not thoroughly diagnose and prevent this disease, eventually leading to severe sequelae by missing the best time point for therapy. In the past decade, various nanoparticles that could selectively respond to ROS have been developed and applied in IRI. These advanced nanomedicines have shown efficient performance in detecting and treating a series of $\mathrm{IRI}$ (e.g., acute kidney injury, acute liver injury, and ischemic stroke, etc.), which are well-summarized in the current review. In addition, the nano-platforms (e.g., anti-IL-6 antibody, rapamycin, and hydrogen sulfide delivering nanoparticles, etc.) for preventing $\mid \mathrm{IRI}$ during organ transplantation have also been included. Moreover, the development and challenges of ROS-responsive nanomedicine are systematically discussed for guiding the future direction.

Keywords: bioresponsive nanomedicine, ischemia-reperfusion injury, reactive oxygen species, organ transplantation, nanoparticles

\section{INTRODUCTION}

Instead of restoring and saving tissues from hypoxic conditions, the reperfusion of blood would cause severe damage to those ischemic organs via oxidative stress, which is referred as ischemiareperfusion injury (IRI). Normally, IRI is strongly associated with a series of diseases, such as the infraction of organs, including stroke, renal infraction, and trauma, etc. Notably, the concern of IRI often affects the best timing of reperfusion, the most effective therapy for treating ischemic disease, for example, the acute myocardial infarction (AMI) (Benjamin et al., 2017). Moreover, the acute myocardial IRI can lead to coronary heart disease (CHD) that accounts for over 366,800 deaths per year and more cases with disability (Benjamin et al., 2018).

In addition to the interruption of cellular homeostasis that could damage cells via apoptotic and autophagic pathways, ischemia-reperfusion would bring great oxidative stress and further injure organs via the generation of redox free radicals, for instance, reactive oxygen species (ROS) (Zhang et al., 2007). Although appropriate ROS is essential for intracellular signaling and immune response against pathogens, the excessive ROS can not be entirely scavenged by cellular antioxidants, which would mediate the severe damage on cells by destroying organelle, cell membrane, and nuclei DNA (Nita and Grzybowski, 2016). To protect injured tissues and prevent further damage of organs during transplantation, the administration of antioxidant reagents for scavenging ROS has been 
widely applied, such as edaravone (Fu et al., 2020). However, the usage of edaravone is generally accompanied by a series of side effects ranging from bruising to skin inflammation or rash, leading to many concerns and demands of new types of antioxidant reagents.

In the comparison of traditional drugs, the novel nanomedicine has been gifted with more functional features including high cargos loading, specific targeting, and in-situ theranostics (Chen et al., 2018a,b; Qiao et al., 2020). With these highly controllable functions, precision therapy could be achieved. As a representative, paclitaxel albumin-bound nanoparticles (Abraxane), the FDA-approved nanomedicine has been widely employed for fighting cancer. More importantly, various nanoparticles demonstrate excellent redox activity by trapping free radicals or self-oxidation (Ni et al., 2019). Notably, an increasing number of nano-antioxidants [e.g., Mo-based polyoxometalate nanoclusters (POM), framework nucleic acid (FNA), hydrophobic ceria nanoparticles (NPs), etc.] were recruited as ROS scavenger in dealing with various diseases associate with IRI (e.g., acute kidney injury, acute liver injury, and ischemic stroke, etc.). Here, those advanced ROS-responsive nanoparticles along with antioxidants delivering nano-platforms, have been summarized in the current review to highlight the recent innovation of nanomedicine in dealing with IRI associated diseases. Moreover, the perspective of future development and facing challenges are systemically discussed.

\section{IRI AND ITS THREATS}

Organs will become ischemic if related vasculars were blockaged, which could induce irreservable damage inside the organs. However, the acute reperfusion for ischemics would cause IRI in some patients, which has been widely observed in various diseases including acute coronary syndrome, hepatic/renal acute injury and organ transplantation (Varadarajan et al., 2004). Eventrually, this unexpected complication, IRI will directly lead to a series pathological changes that manifests in brain edema, infarct progression, hemorrhagic transformation, and worsening neurologic system (Zhai et al., 2013; Prabhakaran et al., 2015). More importantly, the severe IRI may further induce systemic diseases including the inflammatory response syndrome (SIRS) and multiple organ dysfunction syndrome (MODS), and increase the mortality of patients, especially for those from the intensive care unit (Cryer, 2000).

\section{GENERAL STRATEGIES FOR DEALING IRI}

How to deal with the IRI is of clinical importance, therefore arousing the interests of diverse scientific communities. From the aspect of clinical view, three key factors have been focused on improving the efficiency and safety of reperfusion treatment, including developing different generation thrombectomy devices, lowering the time to reperfusion and applying potential imaging techniques for enrolling suitable patients (Prabhakaran et al., 2015). However, the reality of the synergistic role of various biological mechanisms in IRI made it challenging to prevent or treat. Indeed, some medications with predicted benefits to IRI have not been proved with a definite role in prevention or treatment, likely vitamin C (Hill et al., 2019), morphine (Le Corvoisier et al., 2018), etc. In the field of liver transplantation, nearly all randomized clinical trials have ultimately failed to ameliorate liver IRI in patients (Zhai et al., 2013). Therefore, innovative strategies with smart features for targeting local environmental factor, such as ROS would be an exciting direction for IRI treatment in the future.

\section{NANOMEDICINE APPLIED IN IRI THERAPY}

Ischemia could be induced via physical conditions (e.g., elevated blood pressure and diabetes, etc.) or damage, which can further trigger following IRI in various organs, ranging from cerebral to renal system. Notably, the elevated ROS after reperfusion mainly contributes to cellular and tissue damage. Recently, a series of novel nanomedicine (e.g., POM and Ceria NPs, etc.) has been designed for treating affected tissues via the smartly ROSresponsive scavenge and imaging of redox in the ischemic area (Table 1).

\section{NANOMEDICINE FOR CEREBRAL IRI}

Cerebral ischemia that generally caused by stroke is associated with high mortality and disability rate. More importantly, ROS produced by cerebral ischemia-reperfusion can further damage brain tissues via neurodegeneration. To overcome this severe disease, various of nanomaterials including polymer NPs (Reddy and Labhasetwar, 2009; Petro et al., 2016; Ghosh et al., 2017; Mei et al., 2019; Mukherjee et al., 2019), ceria NPs (Zhang et al., 2018), polyoxometalate (POM) nanoclusters (Li et al., 2019a), and Framework Nucleic Acids (Li et al., 2019b) have been applied as protective agents, indicating the high efficiency in saving cerebral tissue via ROS scavenging. Notably, the high biocompatibility allows the polymer to be widely recruited for delivering antioxidant reagents [i.e., superoxide dismutase (Reddy and Labhasetwar, 2009), antioxidants catalas (Petro et al., 2016), and curcumin (Mukherjee et al., 2019), etc]. Besides, a dual functional polymer NPs, t-PA@iRNP was successfully employed to induce thrombolytic and antioxidant therapies in cerebral tissue (Mei et al., 2019). With the encapsulation of tissue plasminogen activator ( $\mathrm{t}-\mathrm{PA}$ ) and conjugation of 4amino-2,2,6,6-tetramethylpiperidine-1-oxyl (4-amino-TEMPO), self-assemble t-PA@iRNP ( $\sim 50 \mathrm{~nm})$ demonstrated an acidictriggered $(\mathrm{pH}=6.2)$ thrombolytic activity and a significant decrease of ROS production in middle cerebral artery occlusion (MCAO) model mice. More importantly, the antioxidant effect of t-PA@iRNP efficiently avoided the subarachnoid hemorrhage induced by t-PA, releasing a great potential of this dual therapy via synergistic effect.

In addition, novel bio-responsive nanomaterials containing the different status of metal ion (i.e., $\mathrm{Mo}^{5+}$ and $\mathrm{Mo}^{6+}$ ) or oxygen-sensitive bio-structure (i.e., DNA) showed excellent capabilities as antioxidants (Li et al., 2019a,b). For instance, a 
TABLE 1 | The representatives of novel nanomedicine for treating various IRI via ROS savaging.

\begin{tabular}{|c|c|c|c|c|c|c|}
\hline Application & Type of materials & Nanomedicine & Size $(\mathbf{n m})$ & Mechanism & Investigation & References \\
\hline Cerebral IRI & FNA & aC5a-FNA & 10 & $\begin{array}{l}\text { Antioxidant } \\
\text { (FNA)/C5a-blocking }\end{array}$ & Protection for cerebral I/R & Li et al., 2019b \\
\hline Cerebral IRI & Polyoxometalate & POM & $\sim 1$ & Antioxidant & Protection for cerebral I/R & Li et al., 2019a \\
\hline Cerebral IRI & Polymer & t-PA@iRNP & $48 \pm 2$ & $\begin{array}{l}\text { Antioxidant (LMW } \\
\text { nitroxide)/Thrombolysis }\end{array}$ & $\begin{array}{l}\text { Protection for Middle cerebral } \\
\text { artery occlusion }\end{array}$ & Mei et al., 2019 \\
\hline Cardiac IRI & Polymer & PEG-b-PPS & $\sim 100$ & $\begin{array}{l}\text { Antioxidant (ginsenoside } \\
\text { Rg3) }\end{array}$ & Protection for Myocardial IRI & Li et al., 2020 \\
\hline Cardiac IRI & Polymer & CLP NPS & $150 \pm 18$ & $\begin{array}{l}\text { Enhanced FL signal via } \\
\text { oxidative stress }\end{array}$ & IVIS imaging of Myocardial IRI & Ziegler et al., 2019 \\
\hline Cardiac IRI & Polymer & MCTD-NPs & 95 & Antioxidant (TPP) & Protection for Myocardial IRI & Cheng et al., 2019 \\
\hline Hepatic IRI & Ceria & Ceria NPs & $4.48 \pm 0.8$ & Antioxidant & Protection for Partial hepatic IRI & Ni et al., 2019 \\
\hline Hepatic IRI & Ceria & $\mathrm{CeO}_{2}$ & $10-30$ & Antioxidant & Protection for Partial hepatic IRI & Manne et al., 2017 \\
\hline Hepatic IRI & Polymer & BRNPs & 100 & Antioxidant (Bilirubin) & Protection for Partial hepatic IRI & Kim et al., 2017 \\
\hline Hepatic IRI & Polymeric & PVO & 550 & $\begin{array}{l}\text { Antioxidant (peroxalate } \\
\text { esters)/Gas generation }\end{array}$ & $\begin{array}{l}\text { Protection for and US imaging } \\
\text { Partial hepatic IRI }\end{array}$ & Kang et al., 2016 \\
\hline Renal IRI & Carbon QDs & SeCQDs & 40 & $\begin{array}{l}\text { Antioxidant (doping } \\
\text { selenium) }\end{array}$ & $\begin{array}{l}\text { Protection for } \\
\text { Glycerol/Cisplatin-induced AKI } \\
\text { Models }\end{array}$ & Rosenkrans et al., 2020 \\
\hline Renal IRI & FNA & DONs & $90,120,400$ & Antioxidant & $\begin{array}{l}\text { Protection for Glycerol-induced } \\
\text { AKI Model }\end{array}$ & Jiang et al., 2018 \\
\hline Renal IRI & Melanin & MMPP NPS & 4.5 & Antioxidant & $\begin{array}{l}\text { Protection for Glycerol-induced } \\
\text { AKI Model }\end{array}$ & Sun et al., 2019 \\
\hline Cardiac IRI & Silica & DATS-MIONs & $230 \pm 35$ & $\mathrm{H}_{2} \mathrm{~S}$ delivery (DATS) & Protection for Myocardial IRI & Wang et al., 2019 \\
\hline $\begin{array}{l}\text { Organ } \\
\text { transplant }\end{array}$ & Polymer & Anti-IL-6-PLGA-NPs & 100 & Anti-IL-6 delivery & Protection for Heart allograft & Solhjou et al., 2017 \\
\hline $\begin{array}{l}\text { Organ } \\
\text { transplant }\end{array}$ & Micelles & TRaM & $15.3 \pm 2.3$ & Rapamycin delivery & $\begin{array}{l}\text { Protection for Aortic interposition } \\
\text { allografts }\end{array}$ & Zhu et al., 2018 \\
\hline
\end{tabular}

FNA, Framework nucleic acid; DONs, DNA origami nanostructures; FL, Fluorescence; TPP, Triphenylphosphine; LMW, Low molecular weight; IVIS, in vivo fluorescence imaging system; AKI, Acute kidney injury; PEG, Poly ethylene glycoland; PPS, Poly propylene sulfide.

POM nanocluster was developed and employed as an ROSresponsive agent in treating rats with cerebral $\mathrm{I} / \mathrm{R}$ injury ( $\mathrm{Li}$ et al., 2019a). After intrathecal injection, POM could efficiently accumulate in the brain and reduce the oxidative pressure, eventually suppressing the apoptosis of cerebral cells, edema, and infarct volume within the brain (for about 40 50\% decrease). Accompanied by IRI, excessive inflammation plays as another key factor for neuronal damage. Recently, a framework nucleic acids (FNA) conjugating with anti-C5a aptamer (aC5a) was applied in the therapy against IRI in the brain (Figure 1A) (Li et al., 2019b). More specifically, the aC5a-FNA could effectively reduce the $\mathrm{C} 5 \mathrm{a}$ receptor on polymorphonuclear neutrophils (PMNs) and microglia, eventually bringing down the chemotaxis induced by C5a. Meanwhile, the concentrations of C5a (within plasma and penumbra) and detrimental ROS in cerebral issues were well-scavenged by aC5a-FNA administration, which efficiently protects brain cells from IRI, with a much smaller infarct volume of $17.0 \pm 5.6 \%$ in comparison with that in control group $(41.6 \pm 7.1 \%)$.

\section{NANOMEDICINE FOR CARDIAC IRI}

Among the leading causes of death, cardiovascular disease (CVD), especially the myocardial infarction, is extremely dangerous for any patient. As the biggest challenge, the prevention of IRI greatly affects the outcome of therapy for myocardial infarction. To date, several ROS-responsive nanomaterials have been recruited in cardiac IRI therapy via the delivery of antioxidants (i.e., resveratrol and Ginsenoside Rg3, etc.) (Bae et al., 2016; Cheng et al., 2019; Li et al., 2020). This year, a self-assemble nanoparticle poly ethylene glycol-b-poly propylene sulfide (PEG-b-PPS) that smartly responses to ROS was successfully synthesized and used for ginsenoside Rg3 (Rg3) delivery (Li et al., 2020). PEG-b-PPSRg3 exhibited a ROS-dependent release of $\mathrm{Rg} 3$ (80\% release of Rg3 within 1 day when $1.0 \mathrm{mM}$ ROS was presented) and induced antioxidative stress effects by a strong interaction with FoxO3a $(-6.0 \mathrm{kcal} / \mathrm{mol})$. More importantly, the administration of PEG-b-PPS-Rg3 successfully increased the survival rate of $\mathrm{I} / \mathrm{R}$ rat $(90 \%)$ in comparison with the group injected with Free Rg3 (40\%), indicating its high efficiency in responding to and scavenging cardiac-generated ROS. It is notable that precision targeting may increase therapeutic efficiency. To achieve that, Cheng et al. further modified Szeto-Schiller (SS)-31 and ischemic myocardium targeted peptide (IMTP) for preventing myocardial IRI via mitochondria-targeted antioxidant effect (Cheng et al., 2019). As-prepared MCTD-NPs specifically deliver resveratrol into cardiac tissues, reducing 

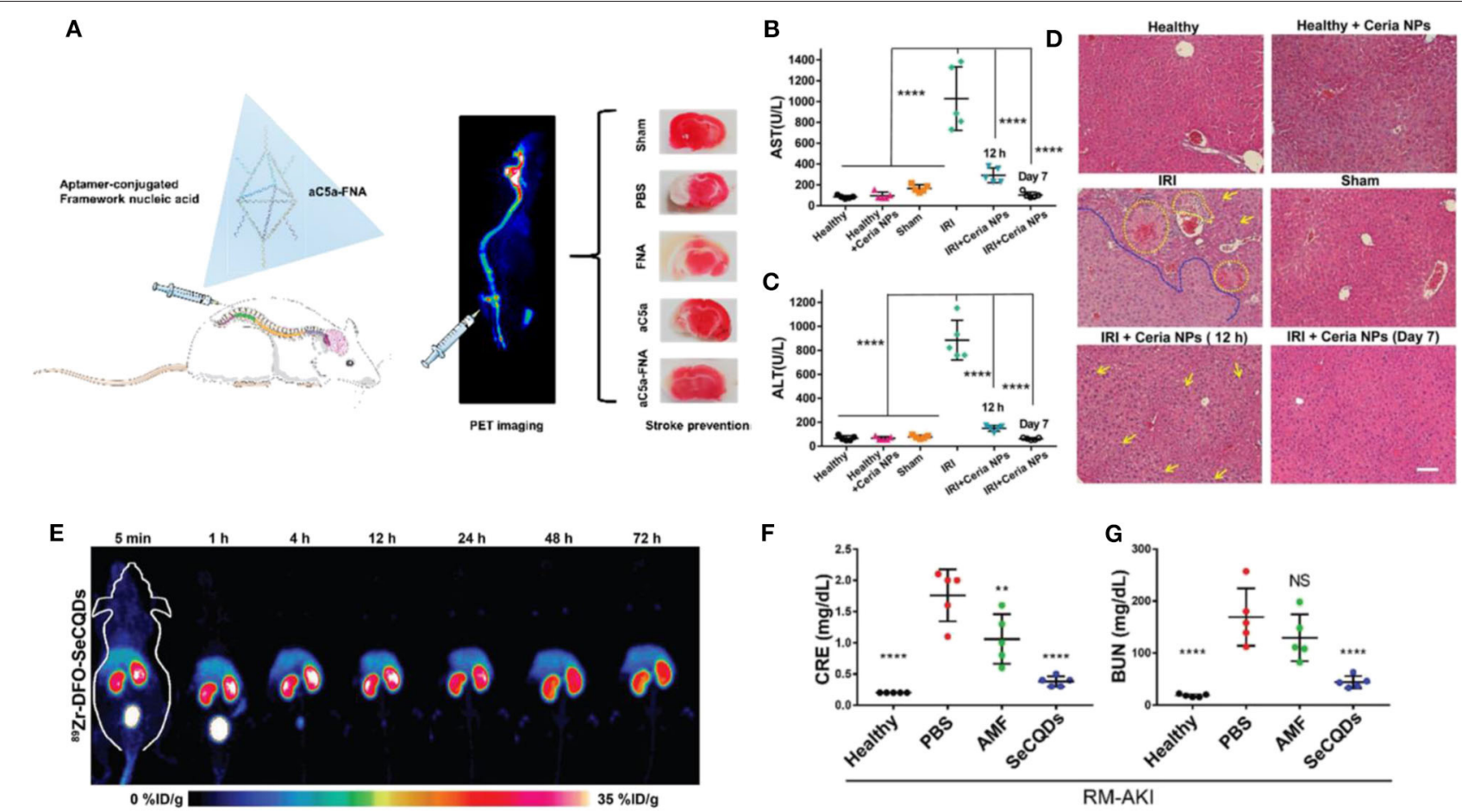

FIGURE 1 | Advanced ROS-responsive nanomedicine for various IRI therapy. (A) The aptamer-conjugated FNA for cerebral IRI therapy. Reproduced with permission from Li et al. (2019b). Copyright 2019, American Chemical Society. The therapeutic effects mediated by Ceria NPs in liver IRI. The level of AST (B) and ALT (C) in serum after different treatments $\left({ }^{\star \star \star \star} p<0.0001\right)$; (D) The HandE staining of liver harvested from different groups. The damage of hepatic cells (i.e., hemorrhage, cytolysis, and necrosis) and severely damaged tissue areas are labeled by yellow and blue dash lines, respectively. Lipid droplets formed are indicated by yellow arrows. Scale bar: $100 \mu \mathrm{m}$. Reproduced with permission from Ni et al. (2019). Copyright 2019, Wiley. The application of SeCQDs in treating AKI for protecting kidney from renal IRI. (E) The time-dependent bio-distribution of ${ }^{89} \mathrm{Zr}$-DFO-SeCQDs in healthy ICR mice via MIP-PET images; The CRE (F) and BUN (G) concentration in serum among groups after various treatments ( ${ }^{\star \star} P<0.01,{ }^{* * \star *} P<0.0001$, NS: no significant difference). Reproduced with permission from Rosenkrans et al. (2020). Copyright 2020, Wiley.

sizes of the infraction and protecting cardiomyocyte from ROS damage.

Notably, the elevated intracellular $\mathrm{Ca}^{2+}$ is strongly associated with the ROS generation in cardiac cells, which provides another therapeutic strategy via the intervention of $\mathrm{Ca}^{2+}$ influx (via anti-L-Type $\mathrm{Ca}^{2+}$ Peptide and CaMKII inhibitor) (Hardy et al., 2015; Wongrakpanich et al., 2017). However, the efficiency of channel blockage is relatively lower. Thus, the combination of antioxidant (i.e., curcumin) reagents and mitochondria-targeting functionalization (i.e., TPP) are required for improving the therapeutic effect.

Besides, several ROS-responsive nano-platforms were also developed for monitoring the status of IRI during the therapy (Yang et al., 2016; Ziegler et al., 2019). Specifically, Peter Lab developed CLP nanoparticles that could smartly light up the ROS-generated myocardium area after ischemic/reperfusion (Ziegler et al., 2019). The presentation of ROS could restore the fluorescent intensity of Ce6 that was attenuated by the aggregation-induced fluorescence quenching. By this strategy, damaged cardiac areas could be real-time detected and monitored throughout the first day after reperfusion.

\section{NANOMEDICINE FOR HEPATIC IRI}

Liver, one of the major organs has played a key role in metabolisms and been involved in various functions, which demands a lot of blood supply. Hepatic ischemia and IRI, a serious complication caused by liver diseases or transplantation would directly trigger irreversible damage in liver tissue. In comparison with other organs, especially the brain, the liver is able to actively "uptake" foreign agents from blood, which allows a series of therapeutic agents to accumulate inside. Thus, antioxidant gene delivery (i.e., expression genes of extracellular superoxide dismutase, catalase or TLR-4 siRNA) (He et al., 2006; Jiang et al., 2011), antioxidant prodrug (i.e., BRAP, etc.) (Lee et al., 2015), and nanoparticles $\left(\mathrm{CeO}_{2}\right.$ and platinum $\mathrm{NP}_{\mathrm{S}}$, etc.) (Katsumi et al., 2014; Kang et al., 2016; Li et al., 2016; Kim et al., 2017; Manne et al., 2017; Ni et al., 2019) were recruited in treating herpetic IRI. For example, a nanoparticle was designed to release p-hydroxybenzyl alcohol (HBA) when $\mathrm{H}_{2} \mathrm{O}_{2}$ or LPS was presented, which could remove ROS and decrease the level of pro-inflammation factors (i.e., NO and TNF- $\alpha$ ) (Lee et al., 2015). Moreover, Kang et al. (2016) developed smartly bio-responsive nanoparticles that could provide ultrasound (US) imaging signal 
during $\mathrm{H}_{2} \mathrm{O}_{2}$ scavenge. As-prepared PVO nanoparticles could quickly react with ROS and generate $\mathrm{CO}_{2}$ in liver tissue with IRI, offering detectable US signals as early as 25 min post injection.

Additionally, metal nanomaterials exhibiting high reducibility were used as therapeutic agents for hepatic IRI. Among all, $\mathrm{CeO}_{2} \mathrm{NP}_{\mathrm{S}}$ demonstrated high biocompatibility and efficiency. More specifically, a uniform-sized ceria NPs $(\sim 4.5 \mathrm{~nm})$ was synthesized, containing two valences of $\mathrm{Ce}\left(32.01 \%\right.$ of $\mathrm{Ce}^{3+}$ and $67.99 \%$ of $\mathrm{Ce}^{4+}$ ) ( $\mathrm{Ni}$ et al., 2019). Administration of $\mathrm{CeO}_{2} \mathrm{NP}_{\mathrm{S}}$ greatly attenuated the IRI in liver tissue as early as $12 \mathrm{~h}$ post reperfusion (Figures 1B-D). It is crucial that the ROS scavenge mediated by $\mathrm{CeO}_{2} \mathrm{NP}_{\mathrm{S}}$ efficiently inhibits the pro-inflammation cytokine secretion (i.e., IL-12, IL-1, and TNF- $\alpha$, etc.) and an influx of immune cells (i.e., monocyte and neutrophils). Meanwhile, $\mathrm{CeO}_{2} \mathrm{NP}_{\mathrm{S}}$ also illustrated a high quality in preventing intestinal cells from ROS-based IRI (Gubernatorova et al., 2017).

\section{NANOMEDICINE FOR RENAL IRI}

Acute kidney injury (AKI) that is triggered by diseases or physical damage could lead to renal disorder/failure. In addition to general therapies like dialysis, ROS-responsive nanomedicine may provide another approach for AKI treatment. Mainly, the functional incapacitation of kidney will directly result in passive accumulation of "waste" inside, including nanomedicine injected. By taking this shortcut, advanced nanomaterials with additional functions such as acid-activated (Yoshitomi et al., 2011) or magnetic resonance imaging (MRI) (Sun et al., 2019) were designed for dealing AKI via ROS reduction. Moreover, Minami et al. also synthesized a nanomedicine (e.g., APP-103) that could effectively accumulate in non-functional kidneys and protect them from IRI during AKI or transplantation (Minami et al., 2020).

In comparison, Cai's lab successfully developed two nanomedicines specifically targeting to the kidney. Both nanoparticles, SeCQDs and DONs (DNA origami nanostructures) predominantly accumulated in the health murine kidney with $\sim 10$ and $\sim 27 \% \mathrm{ID} / \mathrm{g}$, respectively, while the kidney after AKI further enhanced the accumulation of nanoparticles (Figure 1E) (Jiang et al., 2018; Rosenkrans et al., 2020). With oxidant-sensitive structures (e.g., doped selenium and nuclei acid), SeCQDs and DONs efficiently removed excessive ROS from renal cells (Figures 1F,G).

\section{ALTERNATIVE STRATEGIES FOR IRI THERAPY AND ORGAN TRANSPLANTATION}

In addition to an active reduction of cellular ROS, cytoprotective reagents such as hydrogen sulfide $\left(\mathrm{H}_{2} \mathrm{~S}\right)$ are also applied as an anti-inflammation agent in various medical situations associated with ischemic diseases. It is notable that various smart nanomedicines have been prepared for $\mathrm{H}_{2} \mathrm{~S}$ delivery, showing promising efficiency (Chen et al., 2019). More specifically, asdesigned mesoporous iron oxide and silica nanoparticles could effectively carry and deliver $\mathrm{H}_{2} \mathrm{~S}$ donor diallyl trisulfide (DATS) to ischemic organs (i.e., heart and aortic graft endothelium), eventually reducing the flowing injury caused by reperfusion (Wang et al., 2016, 2019).

Meanwhile, nano-platforms conjugating with immunemodulators could mediate desirable protection for injured or transplanted organs as well. For instance, the modification of antibodies on PLGA NPs successfully moderated the chronic rejection of transplanted heart by targeting IL-6 secreted by allograft-derived dendritic cells (ADDCs) (Solhjou et al., 2017). Furthermore, the endothelial cell inflammation and pro-inflammatory cytokines secretion following tracheal or aortic allografts could be greatly attenuated by the pre-treatment of rapamycin (Nadig et al., 2015; Zhu et al., 2018).

\section{CONCLUSION/FUTURE DIRECTIONS}

ROS generated by ischemic reperfusion plays a crucial role in the following tissue injury. In other words, this also provides an efficient approach for dealing and preventing IRI via the reduction of ROS. Novel nanoparticles that smartly respond or consume ROS have been designed and proved for their promising performance. However, several directions and challenges should be aware for the future design of ROS-responsive nanomedicine. (a) A combination of multiple functions (i.e., imaging and therapy) in single nanomedicine is highly recommended for monitoring and treating IRI diseases; (b) Desirable IRI therapy may be achieved via dual therapy of ROS scavenge and immunerepress (i.e., $\mathrm{H}_{2} \mathrm{~S}$ delivery); (c) Although alternative strategies, such as antioxidant gene delivery, may provide another direction for IRI therapy, the quick response and antioxidant effects are still the key issues for the future design of ROS-responsive nanoplatforms; (d) Tissue-depended design is strongly recommended as well. For example, the antioxidant capability is more important than tissue targeting in AKI model (e.g., passive accumulation in AKI); (e) Sub-organ/cell-targeting for IRI therapy is highly suggested (i.e., antibody-induced cell targeting). Specifically, the most of antioxidant nanoparticles would be internalized by Kupffer cells during the liver IRI, which may lower the therapeutic effect of nanomedicine in major hepatic cells such as liver sinusoidal endothelial cells; (f) The antioxidant efficiency and biocompatibility should be balanced. Thus, advanced ROSresponsive nanomedicine have shown high-performance in dealing with various IRI, which exhibits a great potential in clinical translation, especially organ transplantation.

\section{AUTHOR CONTRIBUTIONS}

WC planned and wrote this review with the help of DL. All authors contributed to the article and approved the submitted version.

\section{FUNDING}

This work was supported by the National Natural Science Foundation of China (81971668). 


\section{REFERENCES}

Bae, S., Park, M., Kang, C., Dilmen, S., Kang, T. H., Kang, D. G., et al. (2016). Hydrogen peroxide-responsive nanoparticle reduces myocardial ischemia/reperfusion injury. J. Am. Heart Assoc. 5:e003697. doi: 10.1161/JAHA.116.003697

Benjamin, E. J., Blaha, M. J., Chiuve, S. E., Cushman, M., Das, S. R., Deo, R., et al. (2017). Heart disease and stroke statistics-2017 update: a report from the American Heart Association. Circulation 135, e146-e603. doi: 10.1161/CIR.0000000000000485

Benjamin, E. J., Virani, S. S., Callaway, C. W., Chamberlain, A. M., Chang, A. R., Cheng, S., et al. (2018). Heart disease and stroke statistics--2018 update: a report from the American Heart Association. Circulation 137, e67-e492. doi: 10.1161/CIR.0000000000000558

Chen, W., Ni, D., Rosenkrans, Z. T., Cao, T., and Cai, W. (2019). Smart H2Striggered/therapeutic system (SHTS)-based nanomedicine. Adv. Sci. 6:1901724. doi: 10.1002/advs.201901724

Chen, W., Zuo, H., Li, B., Duan, C., Rolfe, B., Zhang, B., et al. (2018a). Clay nanoparticles elicit long-term immune responses by forming biodegradable depots for sustained antigen stimulation. Small 14:1704465. doi: 10.1002/smll.201704465

Chen, W., Zuo, H., Zhang, E., Li, L., Henrich-Noack, P., Cooper, H., et al. (2018b). Brain targeting delivery facilitated by ligand-functionalized layered double hydroxide nanoparticles. ACS Appl. Mater. Interfaces 10, 20326-20333. doi: 10.1021/acsami.8b04613

Cheng, Y., Liu, D.-Z., Zhang, C.-X., Cui, H., Liu, M., Mei, Q.-B., et al. (2019). Mitochondria-targeted antioxidant delivery for precise treatment of myocardial ischemia-reperfusion injury through a multistage continuous targeted strategy. Nanomed. Nanotechnol. Biol. Med. 16, 236-249. doi: 10.1016/j.nano.2018.12.014

Cryer, H. G. (2000). "Ischemia and reperfusion as a cause of multiple organ failure," in Multiple Organ Failure, eds A. E. Baue, E. Faist, and D. E. Fry (New York, NY: Springer), 108-113. doi: 10.1007/978-1-4612-1222-5_12

Fu, Z.-Y., Wu, Z.-J., Zheng, J.-H., Li, N., Lu, J.-Y., and Chen, M.-H. (2020). Edaravone ameliorates renal warm ischemia-reperfusion injury by downregulating endoplasmic reticulum stress in a rat resuscitation model. Drug Des. Devel. Ther. 14:175. doi: 10.2147/DDDT.S211906

Ghosh, S., Sarkar, S., Choudhury, S. T., Ghosh, T., and Das, N. (2017). Triphenyl phosphonium coated nano-quercetin for oral delivery: neuroprotective effects in attenuating age related global moderate cerebral ischemia reperfusion injury in rats. Nanomed. Nanotechnol. Biol. Med. 13, 2439-2450. doi: 10.1016/j.nano.2017.08.002

Gubernatorova, E. O., Liu, X., Othman, A., Muraoka, W. T., Koroleva, E. P., Andreescu, S., et al. (2017). Europium-doped cerium oxide nanoparticles limit reactive oxygen species formation and ameliorate intestinal ischemia-reperfusion injury. Adv. Healthcare Mater. 6:1700176. doi: 10.1002/adhm.201700176

Hardy, N., Viola, H. M., Johnstone, V. P., Clemons, T. D., Cserne Szappanos, H., Singh, R., et al. (2015). Nanoparticle-mediated dual delivery of an antioxidant and a peptide against the L-type $\mathrm{Ca} 2+$ channel enables simultaneous reduction of cardiac ischemia-reperfusion injury. ACS Nano 9, 279-289. doi: 10.1021/nn5061404

He, S. Q., Zhang, Y. H., Venugopal, S. K., Dicus, C. W., Perez, R. V., Ramsamooj, R., et al. (2006). Delivery of antioxidative enzyme genes protects against ischemia/reperfusion-induced liver injury in mice. Liver Transpl. 12, 1869-1879. doi: 10.1002/lt.21001

Hill, A., Clasen, K. C., Wendt, S., Majoros, A. G., Stoppe, C., Adhikari, N. K. J., et al. (2019). Effects of vitamin C on organ function in cardiac surgery patients: a systematic review and meta-analysis. Nutrients 11:2103. doi: 10.3390/nu11092103

Jiang, D., Ge, Z., Im, H.-J., England, C. G., Ni, D., Hou, J., et al. (2018). DNA origami nanostructures can exhibit preferential renal uptake and alleviate acute kidney injury. Nat. Biomed. Eng. 2, 865-877. doi: 10.1038/s41551-0180317-8

Jiang, N., Zhang, X., Zheng, X., Chen, D., Zhang, Y., Siu, L., et al. (2011). Targeted gene silencing of TLR4 using liposomal nanoparticles for preventing liver ischemia reperfusion injury. Am. J. Transpl. 11, 1835-1844. doi: $10.1111 /$ j.1600-6143.2011.03660.x
Kang, C., Cho, W., Park, M., Kim, J., Park, S., Shin, D., et al. (2016). $\mathrm{H} 2 \mathrm{O} 2$-triggered bubble generating antioxidant polymeric nanoparticles as ischemia/reperfusion targeted nanotheranostics. Biomaterials 85, 195-203. doi: 10.1016/j.biomaterials.2016.01.070

Katsumi, H., Fukui, K., Sato, K., Maruyama, S., Yamashita, S., Mizumoto, E., et al. (2014). Pharmacokinetics and preventive effects of platinum nanoparticles as reactive oxygen species scavengers on hepatic ischemia/reperfusion injury in mice. Metallomics 6, 1050-1056. doi: 10.1039/C4MT00018H

Kim, J. Y., Lee, D. Y., Kang, S., Miao, W., Kim, H., Lee, Y., et al. (2017). Bilirubin nanoparticle preconditioning protects against hepatic ischemia-reperfusion injury. Biomaterials 133, 1-10. doi: 10.1016/j.biomaterials.2017.04.011

Le Corvoisier, P., Gallet, R., Lesault, P. F., Audureau, E., Paul, M., Ternacle, J., et al. (2018). Intra-coronary morphine versus placebo in the treatment of acute STsegment elevation myocardial infarction: the MIAMI randomized controlled trial. BMC Cardiovasc. Disord. 18:193. doi: 10.1186/s12872-018-0936-8

Lee, D., Park, S., Bae, S., Jeong, D., Park, M., Kang, C., et al. (2015). Hydrogen peroxide-activatable antioxidant prodrug as a targeted therapeutic agent for ischemia-reperfusion injury. Sci. Rep. 5:16592. doi: 10.1038/srep16592

Li, H., Sun, J.-J., Chen, G.-Y., Wang, W.-W., Xie, Z.-T., Tang, G.-F., et al. (2016). Carnosic acid nanoparticles suppress liver ischemia/reperfusion injury by inhibition of ROS, caspases and NF- $\mathrm{KB}$ signaling pathway in mice. Biomed. Pharmacother. 82, 237-246. doi: 10.1016/j.biopha.2016.04.064

Li, L., Wang, Y., Guo, R., Li, S., Ni, J., Gao, S., et al. (2020). Ginsenoside Rg3-loaded, reactive oxygen species-responsive polymeric nanoparticles for alleviating myocardial ischemia-reperfusion injury. J. Control. Release 317, 259-272. doi: 10.1016/j.jconrel.2019.11.032

Li, S., Jiang, D., Ehlerding, E. B., Rosenkrans, Z. T., Engle, J. W., Wang, Y., et al. (2019a). Intrathecal administration of nanoclusters for protecting neurons against oxidative stress in cerebral ischemia/reperfusion injury. ACS Nano 13, 13382-13389. doi: 10.1021/acsnano.9b06780

Li, S., Jiang, D., Rosenkrans, Z. T., Barnhart, T. E., Ehlerding, E. B., Ni, D., et al. (2019b). Aptamer-conjugated framework nucleic acids for the repair of cerebral ischemia-reperfusion injury. Nano Lett. 19, 7334-7341. doi: 10.1021/acs.nanolett.9b02958

Manne, N. D., Arvapalli, R., Graffeo, V. A., Bandarupalli, V. V., Shokuhfar, T., Patel, S., et al. (2017). Prophylactic treatment with cerium oxide nanoparticles attenuate hepatic ischemia reperfusion injury in sprague dawley rats. Cell. Physiol. Biochem. 42, 1837-1846. doi: 10.1159/000479540

Mei, T., Kim, A., Vong, L. B., Marushima, A., Puentes, S., Matsumaru, Y., et al. (2019). Encapsulation of tissue plasminogen activator in $\mathrm{pH}$-sensitive self-assembled antioxidant nanoparticles for ischemic stroke treatmentsynergistic effect of thrombolysis and antioxidant. Biomaterials 215:119209. doi: 10.1016/j.biomaterials.2019.05.020

Minami, K., Bae, S., Uehara, H., Zhao, C., Lee, D., Iske, J., et al. (2020). Targeting of intragraft reactive oxygen species by APP-103, a novel polymer product, mitigates ischemia/reperfusion injury and promotes the survival of renal transplants. Am. J. Transpl. 20, 1527-1537. doi: 10.1111/ajt.15794

Mukherjee, A., Sarkar, S., Jana, S., Swarnakar, S., and Das, N. (2019). Neuro-protective role of nanocapsulated curcumin against cerebral ischemia-reperfusion induced oxidative injury. Brain Res. 1704, 164-173. doi: 10.1016/j.brainres.2018.10.016

Nadig, S. N., Dixit, S. K., Levey, N., Esckilsen, S., Miller, K., Dennis, W., et al. (2015). Immunosuppressive nano-therapeutic micelles downregulate endothelial cell inflammation and immunogenicity. RSC Adv. 5, 43552-43562. doi: 10.1039/C5RA04057D

Ni, D., Wei, H., Chen, W., Bao, Q., Rosenkrans, Z. T., Barnhart, T. E., et al. (2019). Ceria nanoparticles meet hepatic ischemia-reperfusion injury: the perfect imperfection. Adv. Mater. 31:1902956. doi: 10.1002/adma.201902956

Nita, M., and Grzybowski, A. (2016). The role of the reactive oxygen species and oxidative stress in the pathomechanism of the age-related ocular diseases and other pathologies of the anterior and posterior eye segments in adults. Oxid. Med. Cell. Longev. 2016:3164734. doi: 10.1155/2016/3164734

Petro, M., Jaffer, H., Yang, J., Kabu, S., Morris, V. B., and Labhasetwar, V. (2016). Tissue plasminogen activator followed by antioxidant-loaded nanoparticle delivery promotes activation/mobilization of progenitor cells in infarcted rat brain. Biomaterials 81, 169-180. doi: 10.1016/j.biomaterials.2015.12.009

Prabhakaran, S., Ruff, I., and Bernstein, R. A. (2015). Acute stroke intervention: a systematic review. JAMA 313, 1451-1462. doi: 10.1001/jama.2015.3058 
Qiao, Y., He, J., Chen, W., Yu, Y., Li, W., Du, Z., et al. (2020). Lightactivatable synergistic therapy of drug-resistant bacteria-infected cutaneous chronic wounds and nonhealing keratitis by cupriferous hollow nanoshells. ACS Nano 14, 3299-3315. doi: 10.1021/acsnano.9b08930

Reddy, M. K., and Labhasetwar, V. (2009). Nanoparticle-mediated delivery of superoxide dismutase to the brain: an effective strategy to reduce ischemiareperfusion injury. FASEB J. 23, 1384-1395. doi: 10.1096/fj.08-116947

Rosenkrans, Z. T., Sun, T., Jiang, D., Chen, W., Barnhart, T. E., Zhang, Z., et al. (2020). Selenium-doped carbon quantum dots act as broad-spectrum antioxidants for acute kidney injury management. Adv. Sci. 7:2000420. doi: 10.1002/advs.202000420

Solhjou, Z., Uehara, M., Bahmani, B., Maarouf, O. H., Ichimura, T., Brooks, C. R., et al. (2017). Novel application of localized nanodelivery of anti-interleukin-6 protects organ transplant from ischemia-reperfusion injuries. Am. J. Transpl. 17, 2326-2337. doi: 10.1111/ajt.14266

Sun, T., Jiang, D., Rosenkrans, Z. T., Ehlerding, E. B., Ni, D., Qi, C., et al. (2019). A melanin-based natural antioxidant defense nanosystem for theranostic application in acute kidney injury. Adv. Funct. Mater. 29:1904833. doi: 10.1002/adfm.201904833

Varadarajan, R., Golden-Mason, L., Young, L., Mcloughlin, P., Nolan, N., Mcentee, G., et al. (2004). Nitric oxide in early ischaemia reperfusion injury during human orthotopic liver transplantation. Transplantation 78, 250-256. doi: 10.1097/01.TP.0000128188.45553.8C

Wang, W., Liu, H., Lu, Y., Wang, X., Zhang, B., Cong, S., et al. (2019). Controlledreleasing hydrogen sulfide donor based on dual-modal iron oxide nanoparticles protects myocardial tissue from ischemia-reperfusion injury. Int. J. Nanomed. 14:875. doi: $10.2147 /$ IJN.S186225

Wang, W., Sun, X., Zhang, H., Yang, C., Liu, Y., Yang, W., et al. (2016). Controlled release hydrogen sulfide delivery system based on mesoporous silica nanoparticles protects graft endothelium from ischemia-reperfusion injury. Int. J. Nanomed. 11:3255. doi: 10.2147/IJN.S104604

Wongrakpanich, A., Morris, A. S., Geary, S. M., Mei-Ling, A. J., and Salem, A. K. (2017). Surface-modified particles loaded with CaMKII inhibitor protect cardiac cells against mitochondrial injury. Int. J. Pharm. 520, 275-283. doi: 10.1016/j.ijpharm.2017.01.061

Yang, L., Ren, Y., Pan, W., Yu, Z., Tong, L., Li, N., et al. (2016). Fluorescent nanocomposite for visualizing cross-talk between microRNA-21 and hydrogen peroxide in ischemia-reperfusion injury in live cells and in vivo. Anal. Chem. 88, 11886-11891. doi: 10.1021/acs.analchem.6b03701
Yoshitomi, T., Hirayama, A., and Nagasaki, Y. (2011). The ROS scavenging and renal protective effects of $\mathrm{pH}$-responsive nitroxide radical-containing nanoparticles. Biomaterials 32, 8021-8028. doi: 10.1016/j.biomaterials.2011.07.014

Zhai, Y., Petrowsky, H., Hong, J. C., Busuttil, R. W., and Kupiec-Weglinski, J. W. (2013). Ischaemia-reperfusion injury in liver transplantationfrom bench to bedside. Nat. Rev. Gastroenterol. Hepatol. 10, 79-89. doi: 10.1038/nrgastro.2012.225

Zhang, T., Li, C.-Y., Jia, J.-J., Chi, J.-S., Zhou, D., Li, J.-Z., et al. (2018). Combination therapy with LXW7 and ceria nanoparticles protects against acute cerebral ischemia/reperfusion injury in rats. Curr. Med. Sci. 38, 144-152. doi: 10.1007/s11596-018-1858-5

Zhang, W., Wang, M., Xie, H., Zhou, L., Meng, X., Shi, J., et al. (2007). Role of reactive oxygen species in mediating hepatic ischemia-reperfusion injury and its therapeutic applications in liver transplantation. Transpl. Proc. 39, 1332-1337. doi: 10.1016/j.transproceed.200 6.11 .021

Zhu, P., Atkinson, C., Dixit, S., Cheng, Q., Tran, D., Patel, K., et al. (2018). Organ preservation with targeted rapamycin nanoparticles: a pre-treatment strategy preventing chronic rejection in vivo. RSC Adv. 8, 25909-25919. doi: 10.1039/C8RA01555D

Ziegler, M., Xu, X., Yap, M. L., Hu, H., Zhang, J., and Peter, K. (2019). A self-assembled fluorescent nanoprobe for imaging and therapy of cardiac ischemia/reperfusion injury. Adv. Ther. 2:1800133. doi: 10.1002/adtp.201800133

Conflict of Interest: The authors declare that the research was conducted in the absence of any commercial or financial relationships that could be construed as a potential conflict of interest.

The handling editor declared a past co-authorship with one of the authors WC.

Copyright (c) 2020 Chen and Li. This is an open-access article distributed under the terms of the Creative Commons Attribution License (CC BY). The use, distribution or reproduction in other forums is permitted, provided the original author(s) and the copyright owner(s) are credited and that the original publication in this journal is cited, in accordance with accepted academic practice. No use, distribution or reproduction is permitted which does not comply with these terms. 